\title{
Burgundy from the tropics: Images and references in Brazil / La Bourgogne vue des tropiques : images et références au Brésil
}

\author{
Ivanira Falcade $^{1, \mathrm{a}}$ and Shana Sabbado Flores ${ }^{2, \mathrm{~b}}$ \\ ${ }^{1}$ University of Caxias do Sul (UCS), Master in Biotechnology and Viticultural Manegement, Rua Francisco Getúlio Vargas, 1130, \\ CEP 95.070-560, Caxias do Sul, RS, Brazil \\ ${ }^{2}$ Federal Institute of Education, Science and Technology of Rio Grande do Sul (IFRS) - campus Restinga, Rua Alberto Hoffmann 285 , \\ CEP 91791-508, Porto Alegre, RS, Brazil
}

\begin{abstract}
Relationships between Burgundy and Brazilian wine production are diverse, such as, the use of emblematic vine varieties or the fraternities and the wine tourism routes. The aim of this paper is to analyze how Brazilian experts and broad public perceive Burgundy, in order to identify influences of the Burgundy region in the Brazilian wine industry. The presence and the influence of Burgundy in the Brazilian wine industry were analyzed from an exploratory study based on the perception of experts was made using an electronic structured questionnaire, and publications available to the general public, observed from sites and blogs on the Internet using Google search, with two keywords in Portuguese and French: "Borgonha", "vinho + Borgonha", "Bourgogne" et "vin + Bourgogne". The results were analyzed and organized and it can be observed that, even in different historical and environmental contexts, Burgundy is present in the wine production of Brazil, as a territory of reference and inspiration.
\end{abstract}

\section{Introduction}

La vitiviniculture en Bourgogne et en France est une référence pour des nombreuses régions viticoles dans le monde depuis longtemps. Les relations entre la Bourgogne et la vitiviniculture au Brésil sont diverses, de l'utilisation des cépages emblématiques jusqu'à la création de confréries et des routes œnotouristiques. L'objectif de cette étude a été d'analyser comment les experts et le grand public brésilien perçoivent la Bourgogne viticole, autant que d'identifier des influences de la région bourguignonne à la filière vitivinicole brésilienne.

La vitiviniculture brésilienne a commencé avec les portugais, au $\mathrm{XVI}^{\text {ème }}$ siècle. Les caractéristiques du processus colonial n'ont pas stimulé le développement, ni la constitution des territoires du vin et la vigne est restée une culture domestique, principalement, aux États de São Paulo, de Bahia et de Pernambuco [1]. L'organisation des espaces et la constitution de territoires avec la vigne et le vin comme facteur principal est lié, surtout, à la colonisation italienne, à partir de 1875, comme par exemple dans la région Encosta Superior do Nordeste du Rio Grande do Sul, connu sur le nom de Serra Gaúcha, ainsi que Urussanga et Vale do Rio do Peixe dans l'Etat de Santa Catarina; São Roque et Jundiaí dans l'Etat de São Paulo; Santa Tereza dans l'Etat d' Espírito Santo; Caldas et Andradas dans l'Etat de Minas Gerais, où la vitiviniculture a eu aussi importance dans structure culturalle de ces régions. Tout au long du XXe siècle, plusieurs de ces régions ont vécu des changements territoriaux significatifs et, aujourd'hui, la dynamique économique a des bases

a e-mail : ifalcade@ucs.br

b e-mail : shana.flores@restinga.ifrs.edu.br différentes. Cependant, il y a encore des éléments de la vitiviniculture que se maintient dans la culture et dans l'identité régionale.

Dans les dernières décennies, l'expansion de la culture de la vigne et de la production du vin pour d'autres régions [2] est lié à la disponibilité foncière et/ou comme diversification des investissements, comme dans les cas des régions de la Campanha et de la Serra do Sudeste dans l'Etat du Rio Grande do Sul, le cas du Planalto Catarinense à Santa Catarina, ou de la Vale do São Francisco dans les Etats de Pernambuco et de Bahia. Cette expansion contribue aux processus de réorganisation territoriale de point de vue économique, mais également de point de vue culturel.

La Fig. 1 montre le panorama de la production vitivinicole brésilienne, par Etats et régions principales, en 2013.

\section{L'organisation des territoires de la vitiviniculture bresilienne}

Les activités économiques sont des pratiques sociales qu'occupent, organisent les espaces et structurent les territoires dans lesquels les différents acteurs de la filière établissent et maintient des relations, selon les règles que le pouvoir public a défini ou des règles propres. Cependant, ces pratiques sociales expriment aussi une culture et constituent des liaisons que manifestent l'identité territoriale [3].

Dans ce contexte, la vitiviniculture brésilienne a des éléments qui, en peu plus de 100 ans, ont configuré des territoires et des régions, aux différentes échelles. Jusqu'à la seconde moitié du XIXème siècle, les cépages de Vitis vinifera cultivées étaient principalement

(C) The Authors, published by EDP Sciences. This is an Open Access article distributed under the terms of the Creative Commons Attribution License 4.0 (http://creativecommons.org/licenses/by/4.0/). 


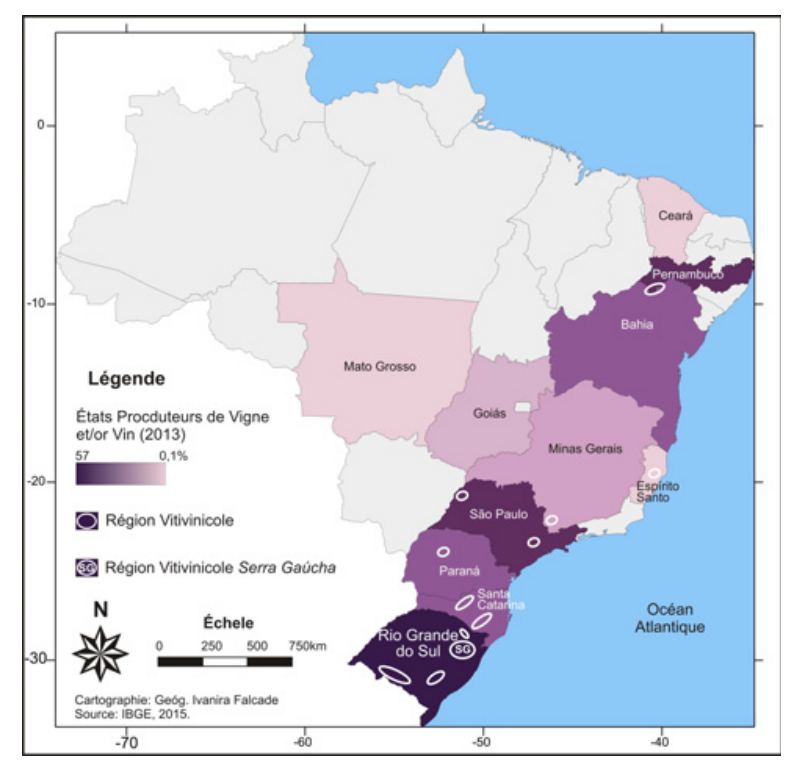

Figure 1. Brésil : vigne et vins, 2013.

d'origine portugaise ou espagnole [4]. La création des centres spécialisés, tels que l'Institut Agronomique de Campinas (São Paulo), en 1887, puis plusieurs Stations Expérimentales de Viticulture et Eonologie, dans la région Serra Gaúcha, dans les années 1920 [5] et de l'Embrapa Uva e Vinho, en 1975, entre autres, ont amené une contribution décisive pour le développement de la viticulture brésilienne. Ces institutions ont fait des recherches pour l'adaptation des cépages de différentes origines, comme Chardonnay, Cabernet Franc, Merlot, cultivées à l'échelle commerciale à partir de la seconde moitié du XXe siècle. Dans les années 1970, se sont installés dans la région de la Serra Gaúcha caves à capital français qui ont augmenté l'influence de la France dans la viticulture brésilienne [6].

L'approche culturelle de la vitiviniculture brésilienne avec la française a d'autres aspects importants, comme l'organisation de confréries et routes œnotouristiques, les études réalisées en France par chercheurs et professeurs, ainsi que la participation aux évènements scientifiques, entre autres. Il y a milliers des agriculteurs qui vivent de la culture de la vigne et des travailleurs dans les caves brésiliennes [7] ; en même temps, il y a centaines de milliers de touristes [8] qui voyagent pour vivre une expérience dans le monde de la vigne et du vin, en visitant des petites régions du Brésil, attirés par son identité culturelle.

L'internet se développe chaque jour, dans la vie quotidienne et aussi dans l'économie, que ce soit les médias, les plateformes de communication ou le commerce électronique. Selon l'IBGE, en 2014, le Brésil était le quatrième pays en nombre de utilisateurs d'internet, c'est-a-dire, plus que $54 \%$ de la population. Le modèle d'exposition des brésiliens est considéré élevé: $76 \%$ des utilisateurs sont à l'internet chaque jour avec 5 heures d'utilisation environ, principalement, pour la recherche des informations et pour loisir avec $67 \%$ des utilisateurs [9].

Ainsi, comment la Bourgogne viticole est vue par les brésiliens?

\section{Matériels et méthodes}

La présence et l'influence de la Bourgogne dans la vitiviniculture brésilienne ont été analysées à partir d'une étude exploratoire, basé sur la perception des experts et des publications disponibles pour le public en général.

La recherche de la perception des professionnels brésiliens liés à la filière viticole a été faite à l'aide d'un questionnaire électronique structuré, avec des questions à choix simples, multiples et des questions ouvertes à réponses courtes. Le questionnaire a été envoyé aux professionnels des institutions de recherche et d'enseignements (qui ont formation superior en œnologie), pour des œnologues de l'Association Brésilienne de Enologie et pour les sommeliers des Associations de Sommeliers de plusieurs Etats. Le questionnaire a été complété par 48 experts [10].

Les informations sur l'opinion du public ont été retirées sur des sites et blogs sur l'internet en utilisant le moteur de recherche Google, en novembre de 2015. La recherche a utilisé deux termes en portugais et deux en français : « Borgonha », «Vinho + Borgonha », «Bourgogne » et 《Vin + Bourgogne ». La première recherche a été faite sans utiliser filtres, en identifiant seulement le nombre de pages par comparaison et, dans un deuxième moment, la recherche a été fait avec l'insertion des filtres selon les critères : 1) pages classifiés comme d'origine au Brésil, 2) en portugais, 3) des années 2014 et 2015 , 4) résultats par pertinence et 5) tous les résultats.

Pour analyser les informations des questionnaires, les données ont été organisées, sur laquellle ont été appliquées des techniques statistiques de traitement de données. Pour la recherche sur l'internet, après l'application des filtres, ont été utilisées les informations des 10 premières pages (ou les pages vues sur le premier écran). Les pages ont été vues et le contenu organisé comme un archive électronique de données textuelles, pour la classification et l'analyse de données. Les principaux résultats sont présentés cidessous.

\section{La Bourgogne vue des tropiques}

La perception sur la Bourgogne serrais présenté en deux moments : d'abord la vision du publique expérimenté dans le domaine de la vigne et vin, appelé les experts, pour après analyser les informations disponibles pour les consommateurs en général, le grand publique.

\subsection{La Bourgogne vue par les experts}

Le questionnaire a été complété par des experts avec un profil diversifié en termes de parcours professionnel et de formation : presque $96 \%$ ont une formation universitaire en 9 différents carrières, tels qu'œnologie, agronomie, gestion, géographie, médecine et sommelier, entre autres. Ces professionnels travaillent, surtout, dans des caves $(46 \%)$ et des institutions d'enseignement $(25 \%)$ et de recherche (13\%), mais aussi dans le secteur de bars et restaurants. La plupart des réponses est provenu des hommes (79\%), entre 30 et 60 ans $(68 \%)$, qui habitent dans l'état Rio Grande do Sul (88\%).

Les experts ont connu la Bourgogne dans des études ou formations $(67 \%)$ et/ou à travers la littérature spécialisée 
(54\%), et la considèrent une référence mondiale; Dijon et Beaune sont les zones les plus visités.

Même si les événements scientifiques ont été la raison le plus indiqué pour les voyages, ces occasions n'ont pas été aussi la raison de mieux connaître la région et ces vins, étant donné seule $4 \%$ des experts ont affirmé avoir utilisé les évènements scientifiques pour une telle finalité. Aujourd'hui les principales sources d'information sur la Bourgogne sont les revues et les livres spécialisées en vitiviniculture $(75 \%)$ et l'internet $(63 \%)$, où les sites de recherche sont plus utilisés (29\%). La plupart des experts $(88 \%)$ ont déjà visité la Bourgogne, dont $50 \%$ plusieurs fois, $68 \%$ avec des visites à plus de 5 propriétés (vignes et caves), avec dégustation et achat de vins (en général, plus de 3 bouteilles). Les experts ont voyagé en Bourgogne, notamment, pour des visites techniques (50 \%), des événements scientifiques (29\%) ou pour étudier $(21 \%)$, même parmi ces qui ont visité la région pour une seule fois.

Pour les experts, les vins de la Bourgogne sont pour les dégustateurs expérimentés. Ils ont utilisé 40 différentes expressions pour caractériser les vins, par exemple, « complexe » et 《 fin » $(11 \%)$, « cher » $(6 \%)$, 《qualité », « personnalité », « minéral » $(4 \%)$ et, aussi, les experts sont d'accord que les vins de la Bourgogne sont élégants $(79 \%)$ et complexes (92\%); par contre $17 \%$ des répondants ne connaissent pas le vin de Bourgogne pour le caractériser. Les experts ont fait référence à 33 vins comme représentatifs de la Bourgogne et le plus mentionnés sont Chablis (15\%), Romanée Conti (11\%), Côte d'Or et Côte de Nuits $(6 \%)$, Beaujolais, Clos-Vougeot, Vosne Romanée et Montrachet (4\%).

Dans la viticulture brésilienne, 92,5\% des experts ont associé les cépages Pinot Noir, Chardonnay et Gammay avec la Bourgogne, mais aussi Aligoté, Pinot Blanc et Cabernet Franc ; $84 \%$ des experts associent à la France les cépages Merlot, Cabernet Sauvignon, Cabernet Franc et Sauvignon Blanc, et mais aussi Chardonnay et Pinot Noir, at autres 5 cépages. Parmi les activités dans la vitiviniculture brésilienne ont été évoqué 12 pratiques associées à la Bourgogne, comme le système de conduite/taille Guyot, la récolte manuelle, la macération carbonique et la maturation en tonneau de chêne.

Pour les experts, la Bourgogne occupe une position en premier plan et de référence dans le contexte international, par son patrimoine vitivinicole et par la notion de « terroir », mais ils ne sont pas d'accord si la Bourgogne c'est une référence pour les brésiliens. Entre les aspects observés, bien que la Bourgogne est liée au œnotourisme $(62,5 \%)$, pour les experts, le principal aspect attaché aux vins est la gastronomie $(83,3 \%)$ et avec les vins de Bourgogne pourraient être « harmonisés » avec la musique classique (46\%), jazz ou blues (42\%).

Entre six images de paysages présentées, les experts ont choisi comme les plus représentatives de la Bourgogne le paysage des vignobles de la Côte D'Or (33\%) et le Clos Vougeot $(25 \%)$, où les aspects d'identités sont les vignobles et le patrimoine construit. Les paysages les plus choisis dans les secondes et troisièmes places ont été les images que présentent un lien fort avec le monde de la vigne et du vin : les Hospices de Beaune et la côte avec le village et la plaine avec ses vignobles (Fig. 2).

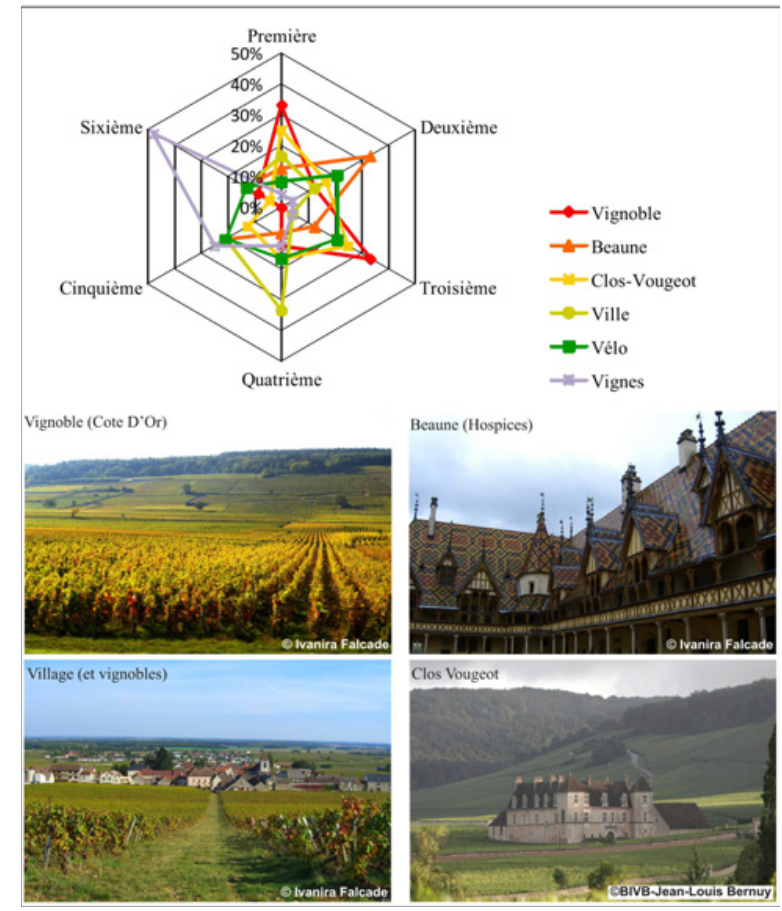

Figure 2. Paysages plus représentatifs de la Bourgogne, selon des experts, 2015. Source des auteurs.

\subsection{La Bourgogne vue par le grand publique}

Le résultat de la recherche sans filtre, sur Google, a identifié 57.200.000 pages pour le mot «Bourgogne » et 564.000 pour le mot « Borgonha », c'est-à-dire, les pages en portugais représentaient $1 \%$ des pages en français. On a compté 7.820 .000 pages pour l'expression 《Vin + Bourgogne » et 182.000 pages pour « Vinho + Borgonha », ce qui correspond à $0,03 \%$ de pages en français. Les résultats ont été ceux attendus, c'est-à-dire qu'il y a beaucoup plus de pages en français, pour le mot « Bourgogne » et l'expression «Vin + Bourgogne », qu'en portugais.

L'application des filtres a permis d'identifier des pages qu'étaient plus liés avec le thème de la recherche, c'està-dire, les informations sur la Bourgogne et ses vins disponibles au grand public. L'usage des critères, de temps et d'origine, a permis l'identification de 20.500 pages pour 《Bourgogne » et 126.000 pages pour «Borgonha »; c'est-à-dire, les résultats ont montré six fois plus des pages indexées en portugais que des pages indexées en français, au Brésil, en 2014-2015. En comparaison à la première recherche, le filtre a sélectionné $22 \%$ des pages pour le mot « Borgonha », mais a sélectionné seulement 0,04\% pour le mot «Bourgogne ». La recherche a identifié 2.330 pages avec l'expression 《Vin + Bourgogne » et 90.700 pages pour l'expression 《Vinho + Borgonha », c'est-à-dire, les résultats ont montré 39 fois plus des pages indexées en portugais que des pages indexées en français, au Brésil, en 2014-2015. Par contre, le filtre a séparé presque $50 \%$ des pages en portugais pour l'expression « Vinho + Borgonha ».

La Fig. 3 présente les résultats organisés par terme et classifiés par les catégories 《vigne et vin », « tourisme », 《 commerce du vin », « interprofession », « université » et « autres » et la Fig. 4 présente un schéma avec l'ordre d'occurrence des catégories. Les illustrations montrent 


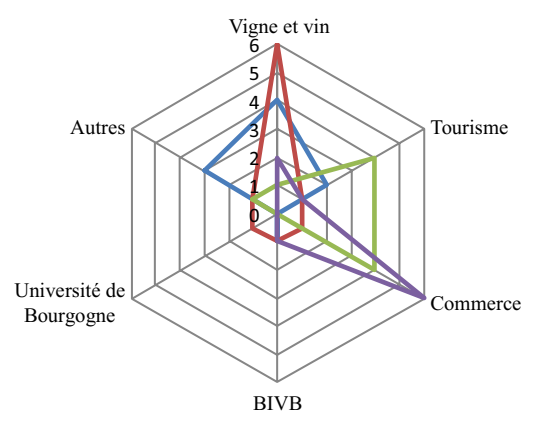

- Borgonha - Vinho + Borgonha - Bourgogne - Vin + Bourgogne

Figure 3. Résultats par catégorie et terme de recherche.

\begin{tabular}{|c|c|c|c|c|}
\hline Ordre & Borgonha & $\begin{array}{c}\text { Vinho + } \\
\text { Borgonha }\end{array}$ & Bourgogne & $\begin{array}{c}\text { Vin }+ \\
\text { Bourgogne }\end{array}$ \\
\hline 1 & Vigne et Vin & BIVB & Tourisme & $\begin{array}{c}\text { Commerce } \\
\text { du vin }\end{array}$ \\
\hline 2 & Outres & Vigne et Vin & Tourisme & BIVB \\
\hline 3 & Vigne et Vin & Vigne et Vin & Tourisme & $\begin{array}{l}\text { Commerce } \\
\text { du vin }\end{array}$ \\
\hline 4 & Vigne et Vin & Vigne et Vin & $\begin{array}{c}\text { Commerce } \\
\text { du vin }\end{array}$ & Tourisme \\
\hline 5 & BIVB & Vigne et Vin & $\begin{array}{l}\text { Commerce } \\
\text { du vin }\end{array}$ & $\begin{array}{c}\text { Commerce } \\
\text { du vin }\end{array}$ \\
\hline 6 & Vigne et Vin & Vigne et Vin & $\begin{array}{c}\text { Commerce } \\
\text { du vin }\end{array}$ & Vigne et Vin \\
\hline 7 & Tourisme & Vigne et Vin & Outres & Vigne et Vin \\
\hline 8 & Outres & Outres & $\begin{array}{c}\text { Commerce } \\
\text { du vin }\end{array}$ & $\begin{array}{l}\text { Commerce } \\
\text { du vin }\end{array}$ \\
\hline 9 & Tourisme & $\begin{array}{l}\text { Commerce } \\
\text { du vin }\end{array}$ & Tourisme & $\begin{array}{l}\text { Commerce } \\
\text { du vin }\end{array}$ \\
\hline 10 & Outres & $\begin{array}{c}\text { Université de } \\
\text { Bourgogne }\end{array}$ & Vigne et Vin & $\begin{array}{l}\text { Commerce } \\
\text { du vin }\end{array}$ \\
\hline
\end{tabular}

Figure 4. Schéma des résultats de la recherche. Source des auteurs.

qu'il y a différences entre les sites en portugais et en français. Il y a des pages que se répètent (six sur dix) pour la recherche des expressions « Borgonha » et «Vinho + Borgonha », ce qui n'est pas observé pour la recherche en français. Dans la liste de pages sélectionnées par le mot en français il y a aussi des pages en portugais.

Les termes en portugais ont indiqué des pages sur la vigne et le vin en général, tandis que la recherche en français fait plus de références aux pages sur le commerce $\mathrm{du}$ vin et sur le tourisme. Le mot 《Borgonha » est peu utilisé pour la vente des vins au Brésil, la recherche ne présente pas sites de cette catégorie dans le premier écran et seulement 1 site avec l'expression «Vinho+ Borgonha ». Même si la recherche a été menée pour filtrer seulement les pages du Brésil, c'est possible de constater que ce filtre est relatif : les résultats ont présenté deux sites institutionnels, du BIVB et de l'Université de Bourgogne. Ces sites commencent en portugais, mais après se suivent des pages en français. Les sites liés au tourisme, aussi à l'œnoturisme, se trouvent avec tous les motsclés de la recherche, mais sont plus fréquent pour le mot « Bourgogne ».

L'analyse des images dans chaque site trouvé dans la recherche, en synthèse, montre des paysages avec des vignobles, du patrimoine construit et aussi des bouteilles de vin, dans le site de commerce. Ce sont des images de l'identité de la Bourgogne (vins, patrimoine et paysages), que les publications électroniques identifient avec la vitiviniculture et la région de la Bourgogne.

\section{Conclusions}

La Bourgogne, classifié par l'UNESCO comme patrimoine de l'humanité, en 2015, est présente dans la vitiviniculture du Brésil, même dans des contextes historiques et environnementaux différents, et peut être un territoire de référence et d'inspiration. Les repères des inspirations bourguignonnes au Brésil sont mis en évidence, à partir des données primaires et secondaires. L'enquête avec des experts a identifiqué les perceptions sur la Bourgogne et ses vins, bien aussi des pratiques viticoles et des cépages bourguignonnes ou françaises dans la vitiviniculture brésilienne. Et la recherche sur internet a identifié les informations que les brésiliens ont disponible sur la Bourgogne et ses vins.

La perception générale sur le vin de la région de la Bourgogne est que les vins sont complexes, élégants et pour dégustateurs expérimentés. La vitiviniculture brésilienne retrouve un lien avec la viticulture bourguignonne, en ce qui concerne les cépages emblématiques et aussi le système de conduite et taille Guyot. Les paysages choisis par les experts comme significatifs pour représenter la Bourgogne sont similaires à ceux trouvés dans les sites analysés.

Egalement, il ressort l'importance de l'internet comme source d'informations, aussi bien pour les experts comme pour le public. Les pages en portugais sur les vins et la Bourgogne peuvent indiquer que le public brésilien utilise plus sa langue pour obtenir des informations et aussi pour les diffuser. Cela peut être pertinent pour les stratégies de communication en ligne, et aussi pour renforcer les liens des brésiliens avec la Bourgogne et ses vins.

\section{Références}

[1] S. Inglês de Sousa. Uvas para o Brasil. Piracicaba, ESALQ (1996)

[2] I. Falcade. Geografía de la vitivinicultura brasileña. In: A. Beretta C. [dir.]. La vitivinicultura uruguaya en la región (1870-2000). Montevideo, Universidad de la República, p.271-297 (2010)

[3] I. Falcade. Le paysage comme représentation spatiale: le paysage viticole comme symbole des indications géografiques des régions Vale dos Vinhedos, Pinto Bandeira et Monte Belo (Brésil). Thèse de doctorat. Porto Alegre/Dijon: UFRGS / UB (2011)

S. S. Flores. Viticulture durable dans le contexte du Brésil: une proposition. Thèse de doctorat. Porto Alegre/Dijon: UFRGS / UB (2015)

S. S. Flores; I. Falcade; R. M. V. Medeiros. Desenvolvimento territorial sustentável sob a perspectiva da vitivinicultura do Rio Grande do Sul. VIII Congresso Latinoamericano da ALASRU. Porto de Galinhas (2010)

[4] S. Inglês de Sousa, Idem (1996)

[5] Cinquentenario della Colonizzazione Italiana nell Rio Grande del Sud 1875-1925, Roma/Porto Alegre: Ministero Degli Affari Esteri/Livraria do Globo (1925) 
E. Pellanda. Aspectos gerais da colonização italiana no Rio Grande do Sul. In: Álbum comemorativo do $75^{\circ}$ aniversário da colonização italiana no Rio Grande do Sul, Porto Alegre, Globo, p. 33-64 (1950)

[6] I. Falcade, Idem (2011)

[7] L. R. Mello et al. Cadastro Vitícola do Rio Grande do Sul. Bento Gonçalves: Embrapa Uva e Vinho/Ibravin (2015)

[8] APROVALE. Disponible en ligne http://www. valedos vinhedos.com.br. Access en 30 nov. 2015

[9] IBGE. PNAD 2014: pesquisa suplementar de acesso à internet e posse de telefone móvel celular para uso pessoal. Disponible en ligne http://www . ibge.gov.br/home/xml/suplemento_pnad.shtm. Access en novembre 2015
IBGE. PNAD 2014: pesquisa suplementar de acesso à internet e posse de telefone móvel celular para uso pessoal. Disponible en ligne http://www . ibge.gov.br/home/xml/suplemento_pnad.shtm. Access en novembre 2015

BRASIL. Secretaria de Comunicação Social da Presidência da República. Pesquisa brasileira de mídia 2015: hábitos de consumo de mídia pela população brasileira. Disponible en ligne $<$ http: // www. secom.gov.br/atuacao/pesquisa/ lista-de-pesquisas-quantitativas-equalitativas-de-contratos-atuais/ pesquisa-brasileira-de-midia-pbm2015.pdf > . Access en novembre 2015

[10] Nous remercions l'attention des experts qui ont répondu le questionnaire 